\title{
Traumatic Acute Subdural Hematoma Localized on the Superior Surface of the Tentorium Cerebelli
}

\author{
-Two Case Reports-
}

\author{
Keigo MATSUmoto, Takashi Houri, Tarumi YAMAKI, and Satoshi UEDA
}

Department of Neurosurgery, Kyoto Prefectural University of Medicine, Kyoto

\begin{abstract}
An 8-year-old boy, who fell downstairs and struck his head, and a 62-year-old female, who hit her head in the automobile accident, presented with unusual traumatic acute subdural hematoma localized on the superior surface of the tentorium cerebelli. Magnetic resonance imaging was useful for determination of the anatomical location of the hematoma, and confirmation of absence of significant parenchymal contusion. Injury of the variant bridging vein possibly caused subdural hematoma over the tentorium.
\end{abstract}

Key words: acute subdural hematoma, head injury, tentorium cerebelli

\section{Introduction}

Acute subdural hematoma following head injury is most frequently located over the convexity of the brain. ${ }^{3,7,12)}$ Acute subdural hematoma in the tentorial region sometimes occurs at birth, ${ }^{4-6)}$ but common head injuries in adults or children resulting in acute subdural hematoma localized on the tentorium are rare. Traumatic tentorial subdural hematoma may not have been recognized as such before the development of magnetic resonance (MR) imaging, since computed tomography (CT) cannot provide detailed evaluation of the deep cranial regions such as the paratentorial region.

We report two cases of traumatic acute subdural hematoma on the superior surface of the tentorium diagnosed by neuroimaging.

\section{Case Reports}

Case 1: An 8-year-old boy fell downstairs and struck his head in the frontal and occipital regions. He was conscious after the accident without marked complaints. Eight hours later, he developed nausea and vomiting and was brought to our hospital. Neurological examination showed nothing abnormal. CT on

Received November 14, 1995; Accepted January 5, 1996

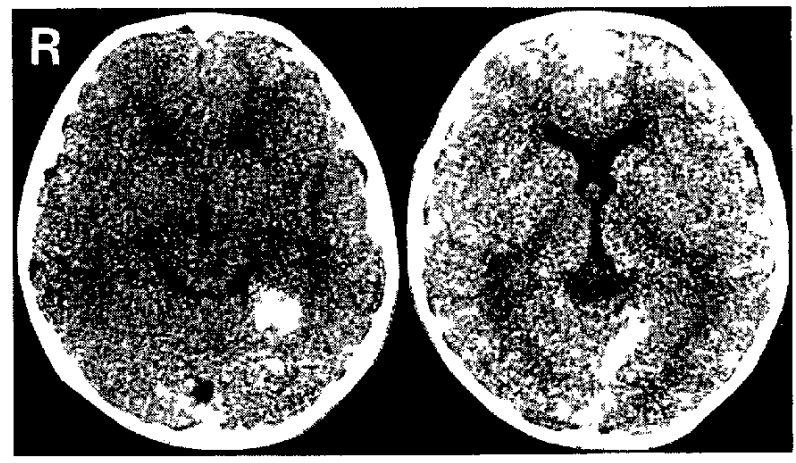

Fig. 1 Case 1. CT scans taken 10 hours after the accident showing hemorrhage in the left temporooccipital region.

admission demonstrated a high density area in the left temporo-occipital region and tentorial edge (Fig. 1). After admission, his condition improved. CT on the 3rd day after the accident showed no enlargement of the hematoma. He was discharged 4 days after admission. MR imaging 1 week after the accident showed a subdural hematoma in the temporo-occipital base on the tentorium cerebelli (Fig. 2). Neuroimaging examinations found no significant hemorrhage in the parenchyma or the cistern.

Case 2: A 62-year-old female was struck on the right frontal region of her head in an automobile accident. 


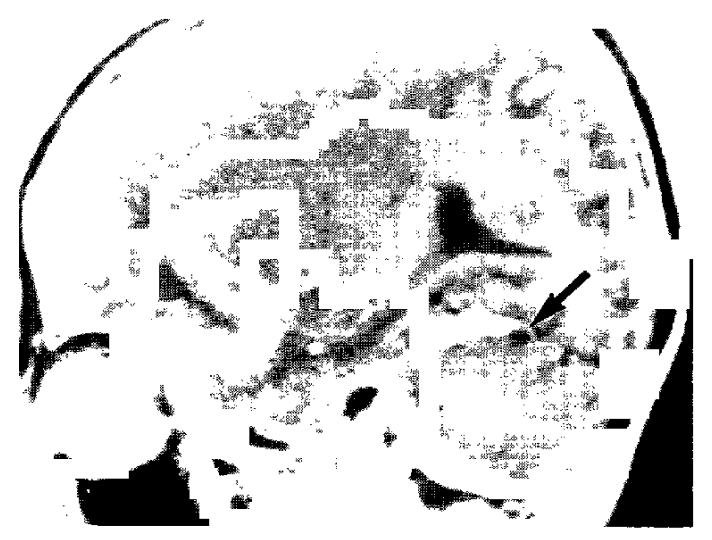

Fig. 2 Case 1. Sagittal $\mathbf{T}_{1}$-weighted MR image taken 1 week after the accident showing a subdural hematoma on the tentorium. The presence of tentorial sinus was suggested as a low signal (arrow) in the posterior margin of the hematoma.

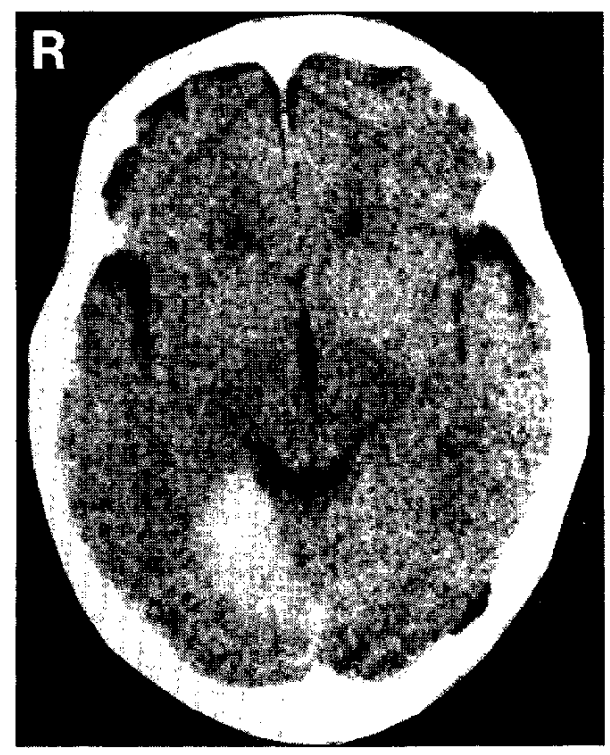

Fig. 3 Case 2. CT scan taken 1 hour after the accident showing hemorrhage in the right tentorial edge.

She was brought to the emergency room in our hospital. She was conscious and had no neurological deficit except for slight amnesia. CT demonstrated a high density area around the right tentorial edge (Fig. 3). She had headache and nausea 1 day later and complained of visual disturbance 3 days later, but these symptoms gradually improved. MR imaging on day 4 revealed a subdural hematoma in the occipital base on the surface of the tentorium but no

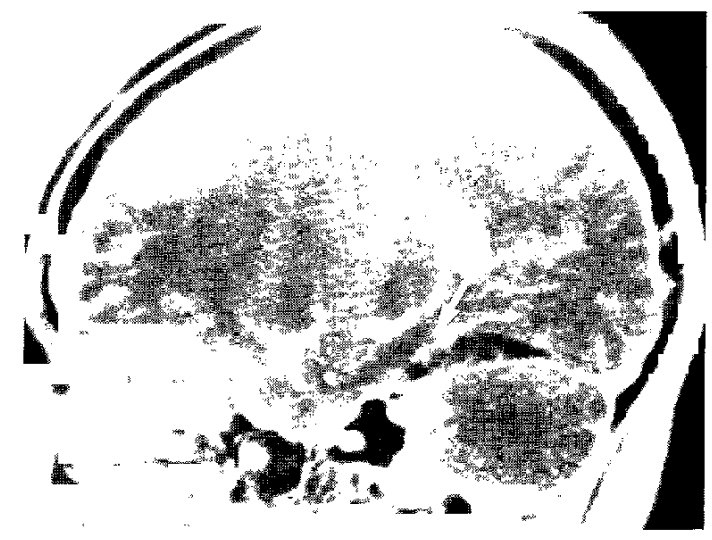

Fig. 4 Case 2. Sagittal $\mathbf{T}_{1}$-weighted $\mathbf{M R}$ image taken 4 days after the accident demonstrating a subdural hematoma on the tentorium. The bridging vein to the tentorium was suggested as a low signal cord-like structure (arrow) crossing the upper margin of the hematoma.

remarkable cerebral contusion adjacent to the hematoma (Fig. 4). No significant hemorrhage was found in the parenchyma or in the cistern. The hematoma became smaller and disappeared completely within 1 month. She was discharged without neurological deficit.

\section{Discussion}

Traumatic subdural hematoma is rarely localized on the superior surface of the tentorium. There are several possible causes: extension of interhemispheric hematoma, tentorial tear, brain contusion, and injury of the bridging vein. Interhemispheric subdural hematoma may extend to the surface of the tentorium, resulting in a similar CT appearance to our cases. ${ }^{2,9)}$ However, we found no hematoma in the interhemispheric region. Subdural hematoma adjacent to the tentorium occurring at birth is believed to be due to tearing of the tentorium itself caused by deformation of the cranium during delivery with or without obstetrical instrumentation. ${ }^{1,4-6)}$ This mechanism is unlikely in our patients since the compliance of the developed cranium is much less than that of the neonate. Neuroimaging detected no significant cerebral contusion in either of our patients. Therefore, these subdural hematomas resulted from tearing of vessels adjacent to the tentorium. From the anatomical view point, the bridging veins to the straight sinus or to the tentorium itself (tentorial sinus) are most likely to be involved. Variations of the basal vein of Rosenthal ${ }^{8,10)}$ 
or the veins draining the temporo-occipital lobe $\mathrm{e}^{11,12)}$ form the bridging veins to the tentorium itself. Matsushima et al. ${ }^{8)}$ reported an incidence of $7.6 \%$ of tentorial sinuses formed by a bridging vein to the tentorial free edge in 26 cadaver hemispheres. Seeger ${ }^{1)}$ found that the pial and dural (tentorial) penetration points of the veins from the temporooccipital cerebra are concentrated in the lateral part of the tentorium. In our patients, MR imaging suggested these bridging veins as a flow void, as a low signal on the tentorium adjacent to the hematoma in Case 1 and a low signal cord-like structure crossing the upper margin of the hematoma in Case 2.

Surgical intervention was not needed in our patients because the hematomas were rather small and the symptoms were slight. Larger hematomas in this region, which may require evacuation, must be evaluated by careful MR imaging to investigate the precise location and detailed surrounding structures of the subdural hematoma. Pathologically, variations of the venous system in the temporo-occipital base might be important for the development of traumatic subdural hematoma in this region, since no parenchymal injuries were seen around the hematoma in our patients.

\section{References}

1) Avraham E, Frishman E, Minz M: CT demonstration of intracranial haemorrhage in term newborn following vacuum extractor delivery. Neuroradiology 35: $107-108,1993$

2) Glista GG, Reichman $\mathrm{OH}$, Brumlik J, Fine M: Interhemispheric subdural hematoma. Surg Neurol 10: 119-122, 1978

3) Gurdjian ES, Webster JE: Head Injuries, Mechanism, Diagnosis and Management. London, Little Brown, 1958, pp 250-267

4) Hayashi $T$, Hashimoto $T$, Honda $E$, Fukuda $S$,
Oshima Y: Neonatal subdural hematoma secondary to tentorial tearing: Clinical analysis on 48 survived cases. No To Shinkei 38: 669-676, 1986 (in Japanese)

5) Hirakawa $K$, Fujimoto $M$, Ueguchi $T$, Naruse $S$ : Acute subdural hematoma in the temporo-occipital base of the newborn due to tentorial tear. No Shinkei Geka 7: 735-742, 1979 (in Japanese)

6) Holland E: Cranial stress in the foetus during labour and on the effect of excessive stress on the intracranial contents; within analysis of eightyone cases of torn tentorium cerebelli and subdural cerebral hemorrhage. Journal of Obstetrics and Gynaecology of the British Empire 29: 549-571, 1922

7) Jamieson KG, Yelland JD: Surgically treated traumatic subdural hematomas. $J$ Neurosurg 37: 137149,1972

8) Matsushima T, Suzuki SO, Fukui M, Rhoton AL Jr, de Oliveira E, Ono M: Microsurgical anatomy of the tentorial sinuses. $J$ Neurosurg 71: 923-928, 1989

9) Okamoto J, Ban $M$, Sakamoto $M$, Takasugi $S$, Matsumoto K: Acute interhemispheric subdural hematoma. Report of a case. No Shinkei Geka 10: 209-213, 1982 (in Japanese)

10) Salamon G, Huang YP: Radiologic Anatomy of the Brain. Berlin, Springer-Verlag, 1976, pp 127-172

11) Seeger W: Microsurgery of Cerebral Veins. Wien, Springer-Verlag, 1984, pp 386-387

12) Stone JL, Rifai MHS, Sugar O, Lang RGR, Oldershaw JB, Moody RA: Subdural hematomas. I. Acute subdural hematoma: Progress in definition, clinical pathology and therapy. Surg Neurol 19: 216-231, 1983

Address reprint requests to: K. Matsumoto, M.D., Department of Neurosurgery, Kyoto Prefectural University of Medicine, Kajii-cho, Kawaramachi-Hirokoji, Kamigyo-ku, Kyoto 602, Japan. 